\title{
Proning in Pregnancies Complicated by Acute Respiratory Distress Syndrome. A Call to Action During the COVID-19 Pandemic.
}

\author{
Liviu Cojocaru ${ }^{1}$, Ozhan Turan ${ }^{1}$, Andrea Levine ${ }^{1}$, Linda Sollecito ${ }^{2}$, Susan Williams ${ }^{2}$, Emad \\ Elsamadicy $^{1}$, Sarah Crimmins ${ }^{1}$, and Shifa Turan ${ }^{1}$ \\ ${ }^{1}$ University of Maryland School of Medicine \\ ${ }^{2}$ University of Maryland Medical Center
}

April 7, 2021

\section{Proning in Pregnancies Complicated by Acute Respiratory Distress Syndrome. A Call to Action During the COVID-19 Pandemic.}

Authors: L Cojocaru, OM Turan, A Levine, L Sollecito, S Williams, E Elsamadicy, S Crimmins, S Turan.

1. Liviu Cojocaru, MD: Division of Maternal-Fetal Medicine. Department of Obstetrics, Gynecology and Reproductive Science. University of Maryland School of Medicine, Baltimore, MD, USA.

2. (lcojocaru@som.umaryland.edu) (ORCID No: 0000-0003-1558-702X).

3. Ozhan M Turan, MD, Ph.D.: Division of Maternal-Fetal Medicine. Department of Obstetrics, Gynecology and Reproductive Science. University of Maryland School of Medicine, Baltimore, MD, USA.

4. (oturan@som.umaryland.edu)

5. Andrea Levine, MD: Division of Pulmonary and Critical Care Medicine. Department of Medicine. University of Maryland School of Medicine, Baltimore, MD, USA.

6. (andrea.levine@som.umaryland.edu)

7. Linda Sollecito, BSN, RN, CCRN: Medical Intensive Care Unit. University of Maryland Medical Center, Baltimore, MD, USA.

8. (lsollecito@umm.edu)

9. Susan Williams, MS, RN, CCRN: Medical Intensive Care Unit. University of Maryland Medical Center, Baltimore, MD, USA.

10. (susanwilliams@umm.edu)

11. Emad Elsamadicy, MD: Division of Maternal-Fetal Medicine. Department of Obstetrics, Gynecology and Reproductive Science. University of Maryland School of Medicine, Baltimore, MD, USA.

12. (eelsamadicy@som.umaryland.edu)

13. Sarah Crimmins, DO: Division of Maternal-Fetal Medicine. Department of Obstetrics, Gynecology and Reproductive Science. University of Maryland School of Medicine, Baltimore, MD, USA.

14. (scrimmins@som.umaryland.edu)

15. Shifa Turan, MD, RDMS: Division of Maternal-Fetal Medicine. Department of Obstetrics, Gynecology and Reproductive Science. University of Maryland School of Medicine, Baltimore, MD, USA.

16. (sturan@som.umaryland.edu)

Correspondence to: Liviu Cojocaru, MD: Division of Maternal-Fetal Medicine. Department of Obstetrics, Gynecology and Reproductive Science, University of Maryland School of Medicine, Baltimore, MD, USA.

(lcojocaru@som.umaryland.edu)

Disclosures: None. 
Acknowledgments: We are grateful to Bill Seiler, Erin Rummel, Kelly Kennedy, Alexandra Bessent, Chris Wells, McLellan Shelby, Jessica Peterson, and Amanda Sullivan for assistance in creating the proning video instructions.

Short title: Proning in pregnancies complicated by ARDS. It's time!

\section{Terminology}

ARDS: acute respiratory distress syndrome

$\mathrm{SpO}_{2}$ : oxygen saturation.

$\mathrm{PaO}_{2}$ : partial pressure of oxygen

$\mathrm{PaCO}_{2}$ : partial pressure of carbon dioxide

$\mathrm{FiO}_{2}$ : the fraction of inspired oxygen

$\mathrm{P} / \mathrm{F}$ ratio: the physiologic relationship between inspired oxygen and the arterial partial pressure, i.e., $\mathrm{PaO}_{2} /$ $\mathrm{FiO} 2$ ratio

PEEP: positive end-expiratory pressure

$\mathrm{L} / \mathrm{m}$ : liters/minute

G: gravida (number of pregnancies)

P: para, i.e., completed pregnancies (term, preterm, aborta, living).

W: weeks.

Hollister: endotracheal tube fastener

Z-Flo: fluidized positioner

A-frame: three fluidized positioners are placed in an "A" shaped frame

\section{Introduction}

Hypoxemia in COVID-19

Silent hypoxemia in COVID-19 was initially described in elderly patients but shortly was reported in younger, previously healthy individuals(1). Although perplexing at first glance, silent hypoxemia can be elucidated through the lens of the basic principles of respiratory physiology(2). In contrast, normocapnia until later stages of the disease is less understood(3). With progressive hypoxemia, increased minute ventilation and cardiac output occur as a compensatory mechanism. As the ventilatory response is limited due to normocapnia, the cardiovascular system becomes the central adaptive mechanism. Its failure to compensate is the proximal cause of tissue injury in profound hypoxemia(4).

The effects of hypoxemia in COVID-19 on the fetus

By and large, acute variation in the oxygen delivery rate does not alter fetal oxygen uptake until its delivery falls to a substantially lower rate(5). This is due to the fetal capacity to increase oxygen extraction and coordinated fetal cardiovascular response(6). Nevertheless, it is essential to remember that the $\mathrm{CO}_{2}$ and $\mathrm{O}_{2}$ gradients allow the diffusion of gases across the placenta. As opposed to unfavorable effects on the mother's compensatory mechanisms, normocapnia favors the fetus, as reduced $\mathrm{CO}_{2}$ alveolar tension increases alveolar $\mathrm{O}_{2}$ tension and prevents the right shift in the oxygen-hemoglobin dissociation curve. These are fundamental physiologic changes in pregnancy that must be accounted for when adjusting ventilatory settings.

\section{Proning considerations in pregnancy}

Prone positioning has been widely utilized in ARDS management before and during the COVID-19 pandemic due to its demonstrated mortality benefits(7). Despite the exponential growth of COVID-19 related 
publications, there remains a paucity of research on proning in ARDS in pregnancy(8), specifically in ARDS secondary to COVID-19(9). Proning in pregnancy requires careful attention to often overlooked physiologic changes in pregnancy and additional technical challenges accompanying a gravid abdomen(10). However, the benefits of proning in pregnancy extend even further than the previously described effects on mortality. When normoxia cannot be maintained in a ventilated pregnant patient, delivery is undertaken even at a preterm gestation. With proning and consequently improved oxygenation, iatrogenic preterm delivery might be avoided.

The purpose of this manuscript is to demonstrate a proning technique that was successfully used at our institution to avoid premature delivery of the fetus while improving maternal outcomes. All technical challenges are addressed in an instructional Video 1 and 2 with a pregnant model with twin gestation at 32 weeks.

\section{Cases}

\section{Data management}

We present a case series of proning in pregnancy complicated by ARDS secondary to COVID-19. The Institutional Review Board at the University of Maryland, Baltimore, approved the initiative (HP-00092582) under COVID-19 and Proning In Pregnancy (COPING) study. We reviewed all the patients' charts with positive SARS-CoV-2 from March 2020 until July 2020 and identified those who developed ARDS. Subsequently, we identified four patients that were proned during the antepartum period. Their medical records were reviewed in detail by the Maternal-Fetal Medicine and Medical Intensive Care Unit clinical team. Data were manually abstracted and entered into the Research Electronic Data Capture hosted at the University of Maryland, Baltimore. Each patient consented to publication for the use of their information for research and education purposes. Moreover, the patients had the opportunity to read their case descriptions.

\section{Search strategy}

MEDLINE was searched from January 1972 until March 2020 using keywords "proning positioning" or "proning position" and "pregnancy". The result did not yield any case series of proning for ARDS in pregnancy. It is possible that manuscripts on ARDS that focused on other interventions had proning as part of the protocol. An example is a case series of extracorporeal membrane oxygenation (ECMO) in pregnancy and postpartum(11). However, we could not identify any case series whose objective was discussion or proning in pregnancy.

\section{Management of ARDS related to COVID-19}

1. Ventilation settings were adjusted, considering physiologic changes of pregnancy. The $\mathrm{SpO}_{2}$ was maintained [?] 94\%(12). The goal for $\mathrm{PaO}_{2}$ was $>70 \mathrm{mmHg}$ and for $\mathrm{PaCO}_{2}<50 \mathrm{mmHg}(12)$. In most cases, the decision for proning was made if the $\mathrm{P} / \mathrm{F}$ ratio was $<150$ on [?] $60 \% \mathrm{FiO}_{2}$ and [?] 10 of $\mathrm{PEEP}(7)$. This should serve as a general rule for initiating and discontinuing proning sessions.

2. The principles of medical treatment are highlighted below and summarized in supplemental Catalog 1. Coverage for superimposed pneumonia was typically provided for patients that required increasing respiratory support. Remdesivir, Canakinumab, Tocilizumab, and convalescent plasma were offered based on inclusion criteria for treatment or clinical trial. Antenatal corticosteroids were administered between 24 and 34 weeks if there was a concern for imminent delivery.

\section{Proning instructions}

Proning in pregnancy requires a methodical approach. We developed and utilized at the University of Maryland Medical Center a standard protocol for proning in pregnancy. Stepwise instructions are illustrated in Video 1. We use a ceiling lift to elevate the patient in the air in our technique with the purpose to alleviates injuries associated with lifting patients. The ceiling lift is not an essential requirement for the procedure. A technique where manual labor was used to prone the patient was previously described, though not on a pregnant (13). 


\section{Results}

During the study period, we identified 100 pregnant patients with SARS-CoV-2 infection. Mechanical ventilation was required in 8 of these patients. In three cases, proning was performed during the antepartum period. The gestational age ranged from 25 and 30 completed weeks of gestation. We briefly described their clinical course below. For each case, a graphical ( Figures 1 and Figure S1-S3) and numerical (Tables S) representation of the ventilatory parameters in relationship with proning timing is available as supplementary material.

\section{Case \#1}

A 32 years old non-Hispanic African American G7P3023 at 24w6d was transferred from an outside hospital (OSH) with acute cholecystitis six days after COVID-19 diagnosis. The patient's past medical history (PMH) was significant for asthma, pernicious anemia, major depressive disorder, morbid obesity, and obstructive sleep apnea. Upon arrival at our facility patient was receiving supplemental oxygen via nasal cannula (NC). The patient underwent laparoscopic cholecystectomy under general anesthesia and remained intubated. During the next three days, the patient's condition worsened and required increased ventilatory support. The patient's family declined Remdesivir or convalescent plasma. Due to deteriorating respiratory condition (increase in PEEP from 12 to 16 and in $\mathrm{FiO}_{2}$ from $50 \%$ to $70 \%$, yielding a $\mathrm{P} / \mathrm{F}$ ratio of 122 ), the patient was proned for 16 hours $(\mathrm{h})$. This resulted in improved oxygenation (decrease in $\mathrm{FiO}_{2}$ to $50 \%$, yielding a $\mathrm{P} / \mathrm{F}$ ratio of 305) (Figure 1). After ten hours of a supine position, the patient was proned again for 14h, which further improved oxygenation (PEEP decreased to 14, and $\mathrm{FiO}_{2}$ increased to 430) (Figure 1). The patient was extubated after nine days of mechanical. Fourteen weeks later, the patient presented in spontaneous labor and had an uncomplicated term vaginal delivery and postpartum course.

Case \#2

A 25 y/o Hispanic G7P3023 was transferred from OSH at 25 weeks with COVID-19 (intubated the day prior to transfer). The patient's PMH was significant for obesity. The following day, the patient underwent bronchoscopy and was soon proned for $16 \mathrm{~h}$ due to worsening respiratory status (increase in PEEP from 12 to 14 and in $\mathrm{FiO}_{2}$ from $50 \%$ to $70 \%$, yielding a $\mathrm{P} / \mathrm{F}$ ratio of 175 ). Following the proning session, the respiratory status improved $\left(\mathrm{FiO}_{2}\right.$ decreased to $40 \%$, resulting in a $\mathrm{P} / \mathrm{F}$ ratio of 340$)$ (Figure S 1). Two days later, Remdesivir was initiated. The patient remained intubated for a total of 7 days. After discharge, the patient's prenatal course was uncomplicated. She underwent induction of labor at 39 weeks of gestation and had an uneventful vaginal birth after cesarean.

Case \#3

A 39 y/o Hispanic G7P4024 with PMH of obesity and asthma presented at 27w6d admitted with COVID-19 respiratory symptoms. Patient oxygen requirement escalated quickly, and intubation was performed less than 48 hours after the presentation. On day five of mechanical ventilation patient self-extubated and was re-intubated. On day seven, the patient was proned for 16 hours due to worsening respiratory status (while less evident on arterial blood gasses, the patient had intermittent $\mathrm{SpO}_{2}$ drops to $80 \%$, requiring temporary increases in $\mathrm{FiO}_{2}$ to $100 \%$ ). Upon supination, the $\mathrm{P} / \mathrm{F}$ ratio dropped from 258 to 155 . However, no further proning was necessary as the respiratory status improved ( $\mathrm{P} / \mathrm{F}$ ratio $>200$ at $\mathrm{FiO}_{2}$ of $40-45 \%$ ) (Figure $\mathrm{S} 2$ ). Two days later, during a sedation holiday, the patient again self-extubated. The resultant prolonged acute hypoxia was complicated by fetal bradycardia and an emergent bedside cesarean delivery at $29 \mathrm{w} 2 \mathrm{~d}$. The patient was extubated on postoperative day four and discharged home on day seven.

\section{Case \#4}

A 32 years old non-Hispanic white G5P2022 at 29 weeks transferred from an OSH with severe COVID-19. The PMH was significant for obesity and diabetes mellitus. During the first three days, the patient was managed on a high-flow nasal cannula and CPAP. Remdesivir was initiated, and the patient was enrolled in the Canakinumab trial (NCT04362813). During the next two days, the patient self-proned three times for $7.3,8$, and $1 \mathrm{~h}$. The instructions for self-proning can be seen in Video 2. The last self-proning session 
was discontinued, and the patient intubated as the $\mathrm{SpO} 2$ was below the goal despite being on maximum non-invasive support (high flow $\mathrm{NC}$ at $\mathrm{FiO}_{2}$ of $100 \%$ providing $40 \mathrm{~L} / \mathrm{m}$ with a non-rebreather mask on top at $15 \mathrm{~L} / \mathrm{m})$. Immediately after intubation, the patient had transient hypoxia episodes with $\mathrm{SpO}_{2}$ dropping to $70 \%$. A gradual recovery was achieved once PEEP was increased to 16 and $\mathrm{FiO} 2$ to $100 \%$. The patient's respiratory status remained precarious $\left(\mathrm{P} / \mathrm{F}\right.$ ratio of 147 at $\mathrm{FiO}_{2}$ of $\left.80 \%\right)$. This prompted proning $(16 \mathrm{~h} 45 \mathrm{~m})$, which improved oxygenation $\left(\mathrm{P} / \mathrm{F}\right.$ ratio of 293 at $\mathrm{FiO}_{2}$ of $\left.60 \%\right)$. The following day the patient was proned (16h) due to intermittent desaturations to 80 . The second session also resulted in improved oxygenation (P/F ratio of 371 at $\mathrm{FiO}_{2}$ of $60 \%, \mathrm{SaO} 2$ [?] 94\% was maintained as well) (Figure $\mathrm{S} \mathrm{3.1).} \mathrm{Concomitant}$ with the second proning session, the patient developed new high-grade febrile morbidity. The following day patient started again to have intermittent episodes of desaturation, responsive to temporary increases in PEEP to 16 and $\mathrm{FiO}_{2}$ to $100 \%$. With a declining $\mathrm{P} / \mathrm{F}$ ratio $\left(151\right.$ on $\left.\mathrm{FiO}_{2} 60 \%\right)$ patient was proned for the $3^{\text {rd }}$ time $(15 \mathrm{~h} 45 \mathrm{~m})$. Once returned to the supine position, the patient was desaturating, requiring the FiO2 to be increased to $80 \%$. The decision was made to re-prone earlier $(16 \mathrm{~h} 15 \mathrm{~m})$. Oxygenation improved $(\mathrm{P} / \mathrm{F}$ ratio increased to 232 at $\mathrm{FiO}_{2}$ of 40\%), and desaturation episodes ceased (Figure S 3.2). Sputum cultures grew a multidrug-resistant Acinetobacter Baumannii that was successfully treated with a combination of high dose antibiotics. The patient was extubated five days after the last proning session. The total hospital length was 23 days. One month after discharge patient had an uncomplicated term repeat cesarean delivery.

\section{Discussion:}

\section{Interpretation of the results}

Similar to their non-pregnant counterparts, pregnant patients with ARDS related to COVID-19 experience benefit in the use of prone positioning. For a syndrome with few therapies available, proning becomes a crucial maneuver that benefits the mother and the fetus by avoiding an iatrogenic preterm delivery during worsening respiratory status.

To our knowledge, this is the first manuscript presenting a detailed description of proning in pregnancy that is supported by institutional experience. Moreover, we had a uniform multidisciplinary approach to the management of ARDS in pregnant patients with COVID-19. In the instructional Video 1, we demonstrate how the challenges of proning in pregnancy are easily overcome when performed by a well-trained staff, even at late gestation. The latest gestation at which proning was described in a critically ill patient is 34 weeks in singleton(14). However, studies in healthy individuals that evaluated maternal-fetal hemodynamic parameters are described as far as 37 weeks(15). None of our patients that were proned suffered any complications during proning.

The limitations of our findings are inherited from the sample size and retrospective nature. While these limitations might restrict the generalizability, it is important to weigh the risks of an intervention or treatment against the risk of withholding its benefits, as "protection by exclusion" of pregnant women from research and therapeutic interventions ultimately might negatively affect their health(16).

\section{Concerns and complications}

\section{Airway challenges in pregnancy}

Pregnancy carries an eightfold increased risk of failed intubation(17). The main reason is the increased vascularity and edema of the respiratory mucosa(18). Therefore, a smaller size endotracheal tube should be used. In addition, due to increased $\mathrm{O}_{2}$ consumption in pregnancy and decreased oxygen reserve, a more precipitous drop in $\mathrm{PaO}_{2}$ is anticipated during intubation(18). Optimal pre-oxygenation without hyperventilation will minimize the magnitude of desaturation. An obstetrically trained anesthesiologist should perform the intubation. It is the standard practice at our institution that they are present for non-emergent intubations outside the obstetrical care unit. The availability of an obstetrically trained anesthesiologist offers the necessary support for airway management during proning, eliminating the hesitation to prone a pregnant patient.

Hemodynamic changes 
Contrary to the common concern of aortocaval compression during the prone position in pregnancy, the prone position provides optimal venous return(19). No clinically significant hemodynamic changes occurred during proning in healthy subjects(15) or those with hypertensive disorders(20).

\section{Fetal monitoring}

Fetal monitoring and obstetrical interventions during the periviable period(21) would depend on institutional policies (e.g., NICU capabilities), fetal factors (e.g., estimated weight), and patient's wishes (or families' desires if periviability counseling has not been performed before intubation). We recommend continuous fetal monitoring past 26 weeks of gestation, which is typically easily achieved during proning (Video 1). A correctly placed tocodynamometer will reliably register uterine contractions. At our institution, a dedicated labor and delivery nurse is assigned to any critically ill patient that requires continuous monitoring.

\section{Consideration for delivery}

In general, delivery should not be performed for the sole reason to improve oxygenation(12). Instead, the delivery decision should balance the risks of neonatal prematurity and the risks of exposing the fetus to intractable maternal hypoxemia. Some authors have suggested a threshold of 28-32 weeks(13, 22). At our institution, we first exhaust maximum ventilatory settings, prone position, and potentially extracorporeal membrane oxygenation. The mode of delivery is based on standard obstetrical indications. Induction of labor in a preterm ventilated patient with COVID-19 has been described(23). Whereas cesarean delivery allows a faster and controlled delivery, it has been associated with significant maternal mortality in ventilated patients(24).

Regardless of the planned mode of delivery, all the supplies necessary for cesarean delivery and neonatal resuscitation should be set up inside the biocontainment unit. A portable neonatal and surgical station in close proximity allows for emergent delivery and neonatal resuscitation if necessary.

\section{Complications related to proning}

We have not experienced any complications related to proning. However, a wide range of complications has been described: airway obstruction, endotracheal tube dislodgment, arrhythmias, loss of venous access, facial and airway edema. It is debatable whether complications with prone positioning are related to lack of familiarity with the procedure rather than proning per se(25). Familiarizing with the procedure and paying particular attention to the endotracheal tube, vascular access, and adequate positioning would result in optimal outcomes. The team should be ready to initiate or escalate vasopressor requirements should hypotension occur, as this can lead to non-reassuring fetal status.

\section{Conclusion}

Among the complexity of managing a pregnant intubated patient, COVID-19 adds additional challenges(26). With multiple treatment strategies going amiss(27), proning remains a well-proven intervention in ARDS and should be considered in pregnant women when indicated. In our case series, we were able to improve the $\mathrm{P} / \mathrm{F}$ ratio while decreasing $\mathrm{FiO}_{2}$ and avoid iatrogenic preterm delivery. Except for the case where the patient self-extubated and required emergent delivery, all patients were successfully extubated, followed for prenatal care, and delivered for usual obstetric indications. We recognize that proning might not be effective in all cases. However, based on its proven benefit in the non-pregnant population(7) and the available evidence in the pregnant population $(8,10,13,14)$, proning positioning is an option to improve oxygenation in patients with severe hypoxemia when the next consideration is delivery of a premature infant or maternal ECMO cannulation.

\section{Disclosure of interests}

The authors disclose no conflict of interests.

\section{Contribution to authorship}

LC and ST drafted the manuscript. All authors were involved in writing and editing of the manuscript. 


\section{Details of patient's consent}

Informed consent was obtained from all patients. Moreover, all patients had the opportunity to review their case descriptions.

\section{Funding}

The study received no funding.

\section{References}

1. Ottestad W, Seim M, Mæhlen JO. COVID-19 with silent hypoxemia. Tidsskr Nor Laegeforen. 2020;140(7).

2. Tobin MJ, Laghi F, Jubran A. Why COVID-19 Silent Hypoxemia Is Baffling to Physicians. Am J Respir Crit Care Med. 2020;202(3):356-60.

3. Cavezzi A, Troiani E, Corrao S. COVID-19: hemoglobin, iron, and hypoxia beyond inflammation. A narrative review. Clin Pract. 2020;10(2):1271-.

4. Bickler PE, Feiner JR, Lipnick MS, McKleroy W. "Silent" Presentation of Hypoxemia and Cardiorespiratory Compensation in COVID-19. Anesthesiology. 2020:10.1097/ALN.0000000000003578.

5. Carter AM. Factors affecting gas transfer across the placenta and the oxygen supply to the fetus. J Dev Physiol. 1989;12(6):305-22.

6. Giussani DA, Spencer JAD, Hanson MA. Fetal cardiovascular reflex responses to hypoxaemia. Fetal and Maternal Medicine Review. 1994;6(1):17-37.

7. Guérin C, Reignier J, Richard J-C, Beuret P, Gacouin A, Boulain T, et al. Prone Positioning in Severe Acute Respiratory Distress Syndrome. New England Journal of Medicine. 2013;368(23):2159-68.

8. Samanta S, Samanta S, Wig J, Baronia AK. How safe is the prone position in acute respiratory distress syndrome at late pregnancy? Am J Emerg Med. 2014;32(6):687.e1-3.

9. Schnettler WT, Al Ahwel Y, Suhag A. Severe ARDS in COVID-19-infected pregnancy: obstetric and intensive care considerations. Am J Obstet Gynecol MFM. 2020:100120-.

10. Ray B, A T. Prone position ventilation in pregnancy: Concerns and evidence. Journal of Obstetric Anaesthesia and Critical Care. 2018;8:7.

11. Barrantes JH, Ortoleva J, O'Neil ER, Suarez EE, Beth Larson S, Rali AS, et al. Successful Treatment of Pregnant and Postpartum Women With Severe COVID-19 Associated Acute Respiratory Distress Syndrome With Extracorporeal Membrane Oxygenation. ASAIO J. 2021;67(2):132-6.

12. Lapinsky SE. Management of Acute Respiratory Failure in Pregnancy. Semin Respir Crit Care Med. 2017;38(2):201-7.

13. Tolcher MC, McKinney JR, Eppes CS, Muigai D, Shamshirsaz A, Guntupalli KK, et al. Prone Positioning for Pregnant Women With Hypoxemia Due to Coronavirus Disease 2019 (COVID-19). Obstetrics \& Gynecology. 2020;136(2).

14. Kenn S, Weber-Carstens S, Weizsaecker K, Bercker S. Prone positioning for ARDS following blunt chest trauma in late pregnancy. International Journal of Obstetric Anesthesia. 2009;18(3):268-71.

15. Oliveira C, Lopes MAB, Rodrigues AS, Zugaib M, Francisco RPV. Influence of the prone position on a stretcher for pregnant women on maternal and fetal hemodynamic parameters and comfort in pregnancy. Clinics (Sao Paulo). 2017;72(6):325-32.

16. Costantine MM, Landon MB, Saade GR. Protection by Exclusion: Another Missed Opportunity to Include Pregnant Women in Research During the Coronavirus Disease 2019 (COVID-19) Pandemic. Obstetrics \& Gynecology. 2020;136(1):26-8. 
17. King TA, Adams AP. Failed tracheal intubation. Br J Anaesth. 1990;65(3):400-14.

18. Chesnutt AN. Physiology of normal pregnancy. Crit Care Clin. 2004;20(4):609-15.

19. Nakai Y, Mine M, Nishio J, Maeda T, Imanaka M, Ogita S. Effects of maternal prone position on the umbilical arterial flow. Acta Obstet Gynecol Scand. 1998;77(10):967-9.

20. Dennis AT, Hardy L, Leeton L. The prone position in healthy pregnant women and in women with preeclampsia - a pilot study. BMC Pregnancy Childbirth. 2018;18(1):445.

21. Raju TN, Mercer BM, Burchfield DJ, Joseph GF, Jr. Periviable birth: executive summary of a joint workshop by the Eunice Kennedy Shriver National Institute of Child Health and Human Development, Society for Maternal-Fetal Medicine, American Academy of Pediatrics, and American College of Obstetricians and Gynecologists. Obstet Gynecol. 2014;123(5):1083-96.

22. Pacheco LD, Saad AF, Saade G. Early Acute Respiratory Support for Pregnant Patients With Coronavirus Disease 2019 (COVID-19) Infection. Obstetrics \& Gynecology. 2020;136(1):42-5.

23. Slayton-Milam S, Sheffels S, Chan D, Alkinj B. Induction of Labor in an Intubated Patient With Coronavirus Disease 2019 (COVID-19). Obstetrics \& Gynecology. 2020;136(5):962-4.

24. Jenkins TM, Troiano NH, Graves CR, Baird SM, Boehm FH. Mechanical ventilation in an obstetric population: characteristics and delivery rates. Am J Obstet Gynecol. 2003;188(2):549-52.

25. Henderson WR, Griesdale DEG, Dominelli P, Ronco JJ. Does prone positioning improve oxygenation and reduce mortality in patients with acute respiratory distress syndrome? Can Respir J. 2014;21(4):213-5.

26. Burgoyne ME, Elsamadicy EA, Cojocaru L, Desai A. COVID-19 Barriers to Care for Pregnant Patients in Prolonged Isolation. Case Reports in Obstetrics and Gynecology. 2020;2020:8847859.

27. Repurposed Antiviral Drugs for Covid-19 - Interim WHO Solidarity Trial Results. New England Journal of Medicine. 2020.

Figure's legend

Figure 1

Following proning, the $\mathrm{FiO}_{2}$ and the PEEP decreased. Higher $\mathrm{PaO}_{2}$ is seen during proning compared to the supine position.

\section{Supplemental Figure's legend.}

\section{Figure S 1}

Before proning, the $\mathrm{PaO}_{2}$ was decreasing despite increasing the $\mathrm{FiO}_{2}$ and the PEEP. After proning, the $\mathrm{FiO}_{2}$ was reduced, and the patient's respiratory status continued to improve.

\section{Figure S 2}

The reduction in $\mathrm{FiO}_{2}$ was unsuccessful before proning. However, after the patient was proned, the respiratory status continued to improve. The PEEP was not depicted on the graph as it remained unchanged at 12.

\section{Figure S 3.1}

The $\mathrm{FiO}_{2}$ requirements decreased after the first proning sessions and the PEEP after the second. A significant improvement in $\mathrm{FiO}_{2}$ and PEEP continued even after the proning sessions were completed.

\section{Figure S 3.2}

Notice the decrease in FiO2 and PEEP requirement with proning episodes. The improvement was substantial with the $3^{\text {rd }}$ proning episode despite superimposed ventilator-associated pneumonia with multidrug-resistant bacteria. 
TablesEach case has a supplementary table that displays the graphs in a numerical format.

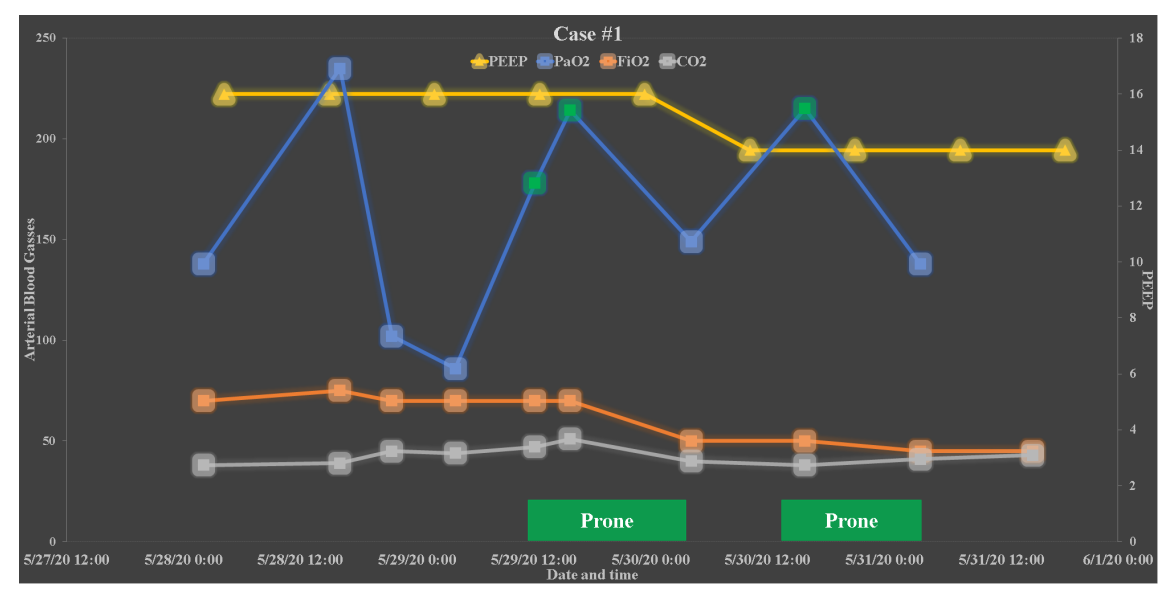

